\title{
Fragments of the Roelofs effect: A bottom-up effect equal to the sum of its parts
}

\author{
ELIZABETH WALTER and PAUL DASSONVILLE \\ University of Oregon, Eugene, Oregon
}

\begin{abstract}
The Roelofs effect is a distortion of perceived space that occurs when a large frame whose center is offset left or right from the objective midline is presented visually to an observer and causes a bias in the observer's subjective judgment of midline. Experiments were designed to test whether an isolated fragment (left or right end) of a Roelofs-inducing frame was capable of generating the Roelofs effect and to determine whether prior experience with intact frames would provide a top-down influence that would bias the Roelofs effect resulting from fragment presentation. Although the fragments did induce an effect, top-down information did not play a significant role even after a 5-day training paradigm. Instead, we found that the effect generated by an intact frame was equal in magnitude to the sum of the effects generated by the individual fragments. In addition, perception was found to be differentially affected by the two ends of the frame, with fragments falling in the right visual field causing a larger effect than those falling in the left.
\end{abstract}

Perception of one's orientation in space is influenced heavily by visual information. In experimental situations, the visual environment can be manipulated to create a misperception of eye level (MacDougal, 1903; Matin \& Fox, 1989), gravitational vertical (e.g., rod-in-frame; Witkin \& Asch, 1948), or self-motion through the environment (Lee \& Aronson, 1974). Visual context can also induce a shift in one's perception of straight-ahead. When an observer is asked to indicate the direction of straightahead in the presence of a large rectangular frame offset from his or her objective midline, the subjective midline is generally reported to be shifted a small amount toward the frame's center (Brecher, Brecher, Kommerell, Sauter, \& Sellerbeck, 1972; Brosgole, 1968; Dassonville \& Bala, 2004b; Dassonville, Bridgeman, Bala, Thiem, \& Sampanes, 2004; Werner, Wapner, \& Bruell, 1953). In turn, this distorted representation of space can lead to a misperception of the frame's egocentric location, with observers underestimating the distance of the frame from their midline (the Roelofs effect; Roelofs, 1935). Similarly, objects contained within the frame appear to observers to be offset in a direction opposite that of the frame (the induced Roelofs effect; Bridgeman, Peery, \& Anand, 1997), since the objects' egocentric locations are judged with respect to the distorted subjective midline (Dassonville \& Bala, 2004b) ${ }^{1}$ For simplicity here, we will use the term Roelofs effect to refer to the misperception of both the frame's location and the locations of the objects enclosed within it. ${ }^{2}$

We thank Mike Posner for his helpful suggestions. This study was partially funded by NIH Systems Physiology Training Grant 5-T32GM07257 to E.W. Correspondence should be addressed to P. Dassonville, Department of Psychology, University of Oregon, Eugene, OR 97403 (e-mail: prd@uoregon.edu).
Previous studies of the Roelofs effect have traditionally used a large rectangular frame as the inducing stimulus, so it might seem that this type of stimulus is required to induce the effect. However, we have seen that a reliable Roelofs effect can also be generated with very different inducing stimuli, some as simple as a small $\left(1^{\circ} \times 1^{\circ}\right)$ distractor placed in the visual periphery (Dassonville, unpublished observations). However, it is not yet clear if the Roelofs effect is driven purely by the bottom-up sensory aspects of the inducing stimulus itself, or whether it might also be mediated by top-down influences. For example, if a small fragment of a larger frame were presented, would the resulting Roelofs effect be similar to that caused by the intact frame that the fragment represents? After all, the brain does regularly fill in portions of the visual scene that are not actually seen (e.g., amodal completion, illusory contours). Here, we hypothesize that observers might be similarly induced to consider a fragment of the frame, namely the left or right edge, as an indicator of the whole frame, thus inducing their brains to automatically fill in the rest. As a result, participants who consider the fragment to be one edge of a partially obscured offset frame should show a midline shift consistent with the normal Roelofs effect. Other participants who have been trained to think of the fragment as part of a centered frame should show no shift of midline. In other words, we tested whether the same fragment might be expected to have differing effects on the perception of midline, depending on how the fragment is mentally completed.

Given this general paradigm, it is also interesting to consider how the effects of individual fragments may combine to yield the eventual Roelofs effect obtained when an intact frame is presented. For example, it is possible that the effect with the intact frame is equal to the sum of the individual effects of the two fragments that combine to make the whole frame. However, other alternatives are 
also possible. The effect of an intact frame could be an average of the effects of the individual fragments. Or perhaps the effect of the intact frame is greater than the sum of its parts, with gestalt laws yielding a more salient perception of the frame when the two ends of the frame are simultaneously presented. It may be that the more peripheral edge (or, alternatively, the more central edge) of the offset frame drives the illusion, leading the corresponding isolated fragment to have a larger influence in the overall Roelofs effect. A second purpose of the studies presented here is to determine how the effects of individual fragments can be combined to achieve the normal Roelofs effect, traditionally induced with an intact frame.

\section{EXPERIMENT 1}

To test for evidence of a top-down effect, we induced a processing bias for our participants by first providing them experience with a particular set of frames, either frames of a single size that were offset to the left or right of the midsagittal plane (offset-frame group) or frames of varying sizes that were centered on the midsagittal plane (centered-frame group). A third group (no-frame group) had no exposure to intact frames before the test trials (Figure 1A). After this initial exposure period, the participants performed a block of trials in which they were asked to move a marker so that it appeared to be straight-ahead in the presence of a fragment depicting the left or right edge of the frame ([-fragment or ]-fragment, respectively), which could appear in one of three horizontal positions. The two sets of intact frames ("offset" and "centered") lined up in such a way that a fragment in a certain position could seem to represent the edge of either an offset or centered frame, depending on the preexposure received (Figure 1B). For example, the rightmost [-fragment (Figure 1B, highlighted fragment) could remind the offset-frame group participants of the right-shifted frame, whereas centered-frame group participants might remember it as the left edge of the small centered frame.

If the Roelofs effect is driven by these high-level representations of the intact frames, the magnitude of the perceptual distortion caused by a fragment should be indistinguishable from that caused by the intact frames to which the participants were preexposed (Figure 1C). The fragments considered to be part of an offset frame should cause a Roelofs effect of the same magnitude as the intact frame, whereas those considered to be part of a centered frame should not cause any Roelofs effect. For example, in the offset-frame group, a purely top-down effect of the rightshifted [-fragment would cause the apparent midline to shift to the right, since the fragment would be considered part of a right-shifted intact frame. However, in the centered-frame group, a right-shifted [-fragment should not cause any shift of the apparent midline, since it would be considered part of a small, centered frame. Finally, in the no-frame group, this same fragment should bias the apparent midline a small distance to the left, since the fragment would be considered an isolated object displaced to the left of midline. To summarize in statistical terms, a purely top-down effect would be indicated by (1) an interaction of fragment location and preexposure group (with significant location effects for the offset-frame and no-frame groups and no location effect for the centered-frame group), and (2) an interaction of fragment polarity (i.e., the left vs. the right end of the frame) and preexposure group (with a significant polarity effect for the no-frame group and no polarity effects for the offset-frame and centered-frame groups).

Conversely, if the fragments are processed in a purely bottom-up manner (with no top-down influence; Figure 1D), the results should be identical regardless of the participants' preexposure: Fragments presented to the left of center should all bias the apparent midline to greater or lesser degrees to the left, depending on frame position, while those presented to the right should drag the apparent midline to the right.

A final option to be considered is the possibility that the Roelofs effect is driven by a combination of top-down and bottom-up influences. This combination would be seen in the data as some weighted average of the two extreme possibilities. Thus, we would expect to see the same patterns of statistical interactions as those described for the purely top-down effects, except that the fragment location effect would be greater than zero in the centered-frame group (albeit significantly smaller than that in the offset-frame and no-frame groups), and the fragment polarity effects would be greater than zero in the offset-frame and centered-frame groups (albeit significantly smaller than that in the noframe group).

\section{Method}

Participants. Seventy-two University of Oregon students who gave informed consent participated in exchange for credit in an undergraduate psychology course. All of the participants were naive to the hypothesis and reported normal or corrected-to-normal vision. Experiment duration was approximately one hour.

Apparatus. Stimuli were presented at eye level to the participants, who sat at a desk in a darkened room. Stimuli were backprojected onto a translucent screen $(128 \mathrm{~cm} \times 96 \mathrm{~cm})$ by a Barco Cine 7 projector at a screen refresh rate of $60 \mathrm{~Hz}$. The participants sat at a comfortable viewing distance away from the screen (approximately $122 \mathrm{~cm}$ ) with their heads steadied by a chin rest. Responses were gathered using a standard computer keyboard (see below).

Stimuli. Centered frames had horizontal extents of either $10^{\circ}, 20^{\circ}$, or $30^{\circ}$ of visual angle, and were centered on the participant's midsagittal plane. Roelofs frames always spanned $20^{\circ}$ and were either centered or offset $5^{\circ}$ to the right or left. The fragments were created by applying a fading gradient to each frame type, leaving a visible portion that extended horizontally $3.5^{\circ}$ from the fully visible edge of the frame before fading to black (Figure 1B). The fading gradient was used intentionally to provide a diffuse rather than an abrupt boundary on the fragment, so that it would appear that a portion of the frame was simply obscured by shadow. Fragments had one of two possible polarities, representing either the left or right edge of the frame (henceforth referred to as [-fragments or ]-fragments, respectively).

Marker circles were $1^{\circ}$ in diameter and could be moved to the right or left using keys on a computer keyboard. Pressing the "F" or "D" keys moved the marker leftward by 1 pixel $\left(0.06^{\circ}\right)$ or 8 pixels $\left(0.45^{\circ}\right)$, respectively; pressing the "J" or "K" keys moved the marker rightward by 1 pixel or 8 pixels, respectively. All stimuli were red, presented on a black background.

Procedure. Each participant completed three blocks of trials. The first block consisted of 72 preexposure trials, in which the participant moved a marker to straight-ahead in the presence of offset frames (offset-frame group), centered frames (centered-frame group), or 
A. Preexposure Groups

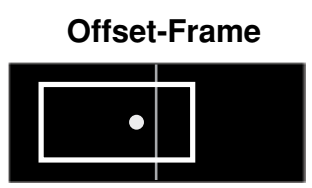

Centered-Frame
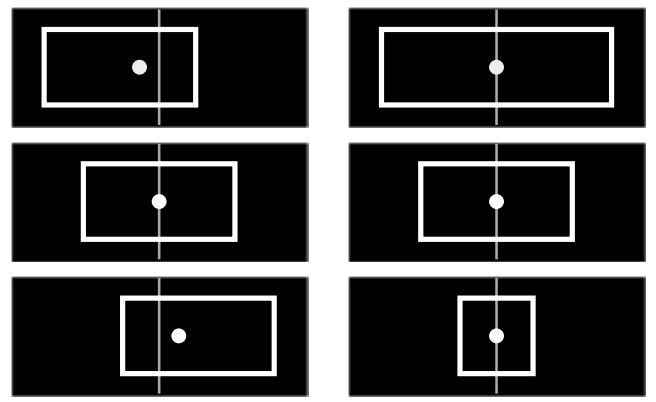

\section{B. Fragment Positions}
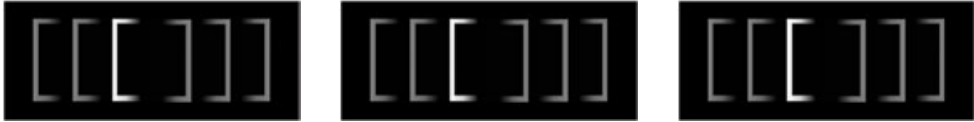

\section{Hypothetical Results (top-down)}
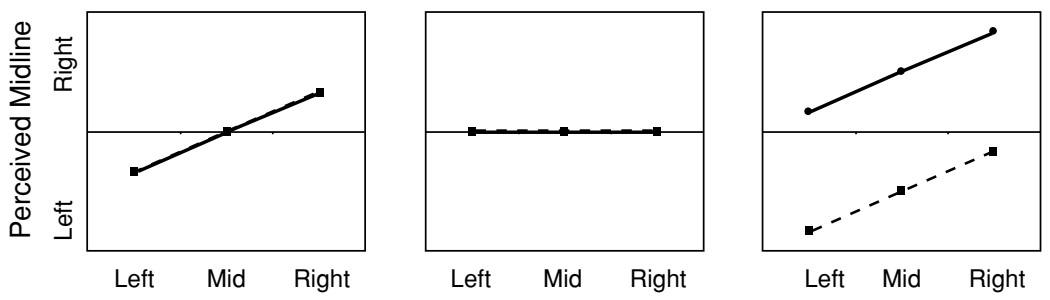

D. Hypothetical Results (bottom-up)
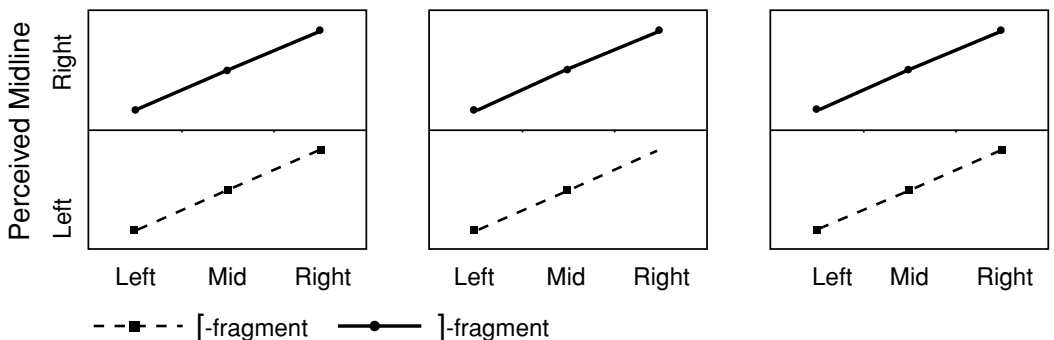

Figure 1. Design and predicted results of Experiment 1. Actual stimuli were red on a black background. (A) Locations and sizes of intact frames in the preexposure block of trials for the three preexposure groups. The vertical line (unseen by participants) depicts the participant's objective midline, with marker circles reflecting distortions of the subjective midline that accompany the offset frames. (B) Possible locations of the [- and ]-fragments. The highlighted fragment depicts the rightmost [-fragment, used as an example in the text; all other possible fragment locations shown in gray. (C) Hypothetical results expected with fragment stimuli if the Roelofs effect is driven by purely top-down mechanisms, with the three plots corresponding to the three preexposure groups. (D) Hypothetical results expected if the Roelofs effect is driven by purely bottom-up mechanisms.

no frames (no-frame group), depending on the preexposure group to which that participant had been assigned. In a second block of 72 trials, all of the participants moved a marker to straight-ahead in the presence of a [-fragment or ]-fragment, which could occupy any of three positions (Figure 1B). In a final block of 72 trials, all of the participants performed the task in the presence of intact offset frames, so that the Roelofs effect could be measured in each participant using the traditional inducing stimuli.

Each trial began with the presentation of a vertical center line. After a keypress to initiate the trial, the screen blanked for $2 \mathrm{sec}$ before a frame (or fragment) appeared, followed $500 \mathrm{msec}$ later by the onset of the marker. Participants had unlimited time to move the marker to straight-ahead, ending the trial with a press of the space bar on the keyboard. The screen then blanked for $1 \mathrm{sec}$ before the start of the next trial.

\section{Results and Discussion}

As expected, results collected in the preexposure block showed a significant effect of frame position for the 
offset-frame group $[F(2,46)=15.87, p<.001]$, indicating the presence of a significant Roelofs effect, and no effect of frame size for the centered-frame group. The data from the fragment block of all 72 participants (Figure 2A) were then subjected to a repeated measures ANOVA of polarity ([-fragment vs. ]-fragment) $\times$ position (left vs. middle vs. right) $\times$ group (offset-frame vs. centered-frame vs. no-frame). A significant main effect of polarity (Table 1) indicated that the apparent midline was generally pulled in the direction of the fragment, and a main effect of position demonstrated that this Roelofs effect was modulated by the location of the fragment. There was also a significant interaction of polarity $\times$ position, with a larger Roelofs effect for the ]-fragments than for the [-fragments (Figure 3). However, this two-way interaction differed across the preexposure groups (as evidenced by a significant interaction of polarity $\times$ position $\times$ group); when tested within the different preexposure groups, the polarity $\times$ position interaction was significant in the offset-frame $[F(2,46)=6.25, p=.01]$ and centered-frame groups $[F(2,46)=12.07, p<.001]$, but not within the no-frame group $[F(2,46)=0.46$, n.s. $]$.

Previous studies have found significant individual differences in susceptibility to visual illusions and performance on spatial tasks in general (see Coren \& Porac, 1987), and in the Roelofs effect specifically (see Dassonville \& Bala, 2004b). A possible confounding factor in the present results is that not all participants demonstrated a significant susceptibility to the Roelofs effect during the final block tested with intact offset frames. Indeed, 25 participants showed no significant Roelofs effect on these trials. It is possible that the inclusion of these less-susceptible participants in our analysis diluted any fragment effects that may have been present in some of the more-susceptible participants. To eliminate this possible confounding factor, we used results from only those participants who showed a significant Roelofs effect in the final block to reanalyze the data. Those participants were distributed across the three groups in roughly equal proportions (offset-frame, $n=15$; centered-frame, $n=16$; no-frame, $n=16$ ). Again, as expected, results from the preexposure block showed a significant effect of frame position for the offset-frame group $[F(2,13)=9.60$, $p<.01]$ and no effect of frame size for the centered-frame group. The fragment block for these participants was subjected to the same repeated measures ANOVA of polarity $\times$ position $\times$ group, as described earlier. Consistent with the previous analysis across all participants, significant effects of polarity, position, and an interaction of polarity $\times$ position (Table 1) were found within this smaller subset of participants. Importantly, however, there was no significant main effect of group, nor any interaction with group.

The lack of polarity $\times$ group and location $\times$ group interactions in the present experiment indicates that the processing of the [- and ]-fragments was not significantly affected by prior experience with offset or centered frames, and thus it appears that the Roelofs effect is driven primarily by bottom-up processes. However, the entire experi-



B Offset-Frame



Centered-Frame

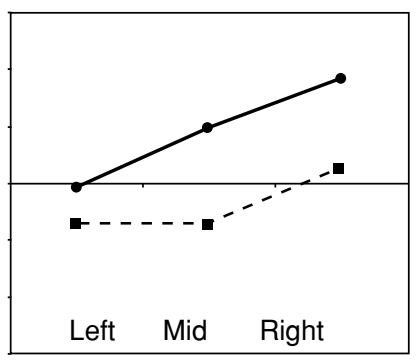

Centered-Frame
Figure 2. Distortions of the perceived midline in each of the preexposure groups of Experiment 1 for the six combinations of fragment polarity and location. (A) Data from all subjects. (B) Data from only those subjects $(n=47)$ with a significant Roelofs effect in the final block of trials (i.e., those tested with intact offset frames). For the data in both $A$ and $B$, there were no significant differences in the processing of the [- and ]-fragments across preexposure groups.

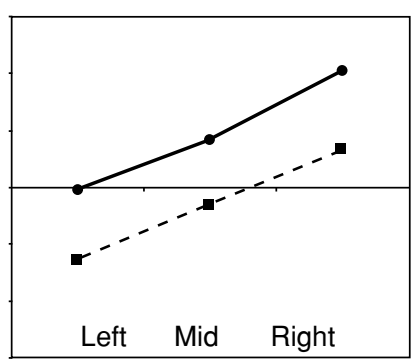

No-Frame




Table 1

Experiment 1: Statistical Results

\begin{tabular}{|c|c|c|c|c|c|c|}
\hline \multirow[b]{2}{*}{ Effect } & \multicolumn{3}{|c|}{$\begin{array}{l}\text { All Subjects } \\
\quad(n=72)\end{array}$} & \multicolumn{3}{|c|}{$\begin{array}{c}\text { Subjects With Significant } \\
\text { Roelofs }(n=47)\end{array}$} \\
\hline & $d f$ & $F$ & $p$ & $d f$ & $F$ & $p$ \\
\hline Polarity ([-fragment vs. ]-fragment) & 1,69 & 58.37 & $<.001$ & 1,44 & 44.02 & $<.001$ \\
\hline Position (left vs. middle vs. right) & 2,138 & 82.55 & $<.001$ & 2,88 & 73.35 & $<.001$ \\
\hline Group (offset- vs. centered- vs. no-frame) & 2,69 & 0.30 & n.s. & 2,44 & 0.06 & n.s. \\
\hline Polarity $\times$ position & 2,138 & 11.25 & $<.001$ & 2,88 & 7.49 & $<.01$ \\
\hline Polarity $\times$ group & 2,69 & 1.19 & n.s. & 2,44 & 1.12 & n.s. \\
\hline Position $\times$ group & 4,138 & 1.07 & n.s. & 4,88 & 2.32 & n.s. \\
\hline Polarity $\times$ position $\times$ group & 4,138 & 3.19 & $<.05$ & 4,88 & 2.29 & n.s. \\
\hline
\end{tabular}

ment took only 1 hour, and it is plausible that top-down effects may require more training and possibly intervening periods of sleep in order for the effects of the training to solidify (see Karni, Tanne, Rubenstein, Askenasy, \& Sagi, 1994). To rule out the possibility that top-down influences have an effect but were not detected here due to inadequate preexposure to the intact frames, an additional set of participants took part in an extended training paradigm over the course of 5 days (Experiment 2). Experiment 3 further explored the significance of the finding that the Roelofs effect driven by the ]-fragments is larger than that driven by the [-fragments.

The results of Experiment 1 also provided an opportunity to examine the manner in which the different components of a frame combine to yield a representation of the whole frame. This can be accomplished by comparing the magnitude of the Roelofs effect for an intact frame with the effects for the two fragments that represent each end of that frame. Thus we devised several hypotheses for how the individual effects of the fragments might be mathematically combined and compared those results with the magnitude of the Roelofs effect that was induced by the intact frames in the third block of trials. Only data from those participants who showed a significant Roelofs effect in the final block of trials with the intact offset frames were considered.
The first hypothesis that was tested (the sum-of-its-parts hypothesis) assumes that the magnitude of the Roelofs effect caused by an intact frame is equivalent to a linear sum of the effects caused by the [- and ]-fragments when each is presented in isolation. The average-of-its-parts hypothesis assumes that the Roelofs effect is equivalent to the average of the effects caused by the corresponding [- and ]-fragments. The outermost-anchor hypothesis assumes that the Roelofs effect is driven solely by the outermost (or most peripheral) end of the frame, such that, for example, a left-shifted frame would have the same magnitude effect as the leftmost [-fragment in isolation. Finally, the innermostanchor hypothesis assumes that the Roelofs effect is driven solely by the innermost (or most central) end of the frame, with, for example, a left-shifted frame having the same magnitude effect as the leftmost ]-fragment in isolation.

We compared the predictions of these four hypotheses to the magnitude of the Roelofs effect induced by the intact frames in the final block of trials for each participant, using paired $t$ tests. The predictions of both the averageof-its-parts and innermost-anchor hypotheses were significantly different from the Roelofs effect induced by the intact frames $[t(46)=5.974, p<.005 ; t(46)=7.705$, $p<.005$, respectively]; thus, we can rule out these hypotheses as explanations of how the individual fragments of the frame combine to yield the representation of an in-

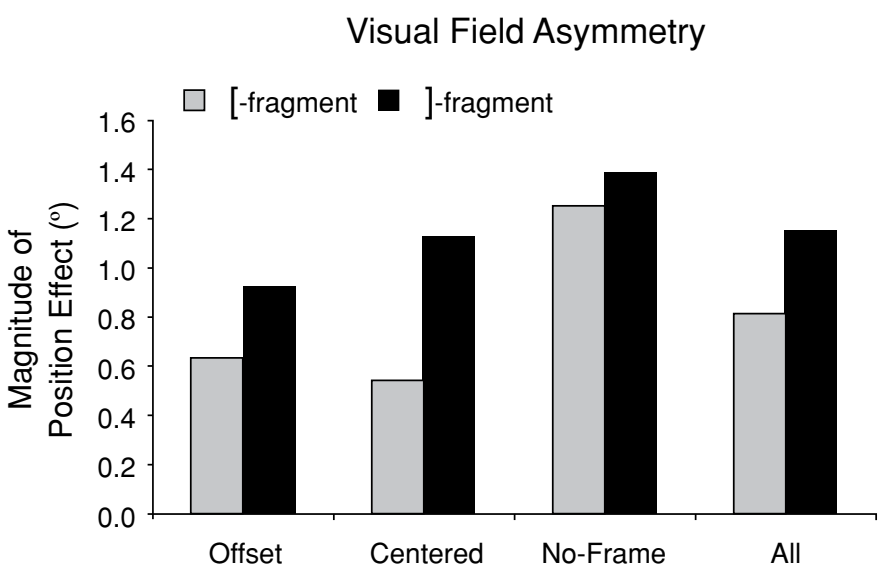

Figure 3. Fragments in the right visual field (]-fragments) caused a greater Roelofs effect than those in the left visual field ([-fragments), as indicated by a significant polarity $\times$ position interaction. 
tact frame. However, we found no significant differences between the magnitude of the Roelofs effect induced with intact frames and that predicted by both the sum-ofits-parts and the outermost-anchor hypotheses $[t(46)<1$, n.s. in both cases], suggesting that either hypothesis could account for the effect. Experiment 3, below, provides additional data that was used to further clarify which of these two hypotheses best accounts for the magnitude of the Roelofs effect induced by intact frames.

\section{EXPERIMENT 2}

The results of Experiment 1 suggest that the Roelofs effect is driven more by bottom-up processing of the incoming visual image than by top-down processes involving a higher level representation of a remembered intact frame. However, we must be cautious in this conclusion, since it was derived only from the null effects of our preexposure paradigm. To help rule out the possibility that these null effects resulted simply from inadequate training, we recruited a second set of participants for an experiment that included a modified preexposure period, and we made a number of changes to ensure that the fragments would be perceived as being part of either centered or offset intact frames. (1) Each preexposure session began with 24 or 48 trials in which an intact frame was briefly presented, after which it dissolved to leave only the fragment. (2) The participant's primary task (the edge task) during the preexposure period was to consider the fragment and then move a marker to indicate the location of the hidden edge of the intact frame. (3) After the participant responded in each trial of the edge task, the entire frame was displayed to provide feedback about the participant's accuracy and to once again reinforce the idea that the fragment was only part of a whole frame. (4) Each preexposure period included a block of trials in which the participants performed the midline task (moving the stimulus so that it appeared to be straight-ahead) in the presence of either fragments or intact frames, presented in random order. (5) Each participant completed five preexposure sessions on consecutive days, which not only provided an increase in the total amount of preexposure, but also allowed for intervening periods of sleep and its accompanying benefits to perceptual learning (Karni et al., 1994; Stickgold, Whidbee, Schirmer, Patel, \& Hobson, 2000). These changes to the preexposure training were made in order to provide the participants every opportunity to associate the fragments with the intact frames. Null effects in this experiment would further suggest that the Roelofs effect is driven by a bottom-up processing of the fragments, rather than a higher level conception of the intact frames.

\section{Method}

Participants. Participants were members of the University of Oregon community who gave their informed consent and were paid for their participation in this experiment. All 8 participants were naive to the hypothesis and reported normal or corrected-to-normal vision. Experiment duration was approximately $1 \mathrm{~h}$ per day for 5 consecutive days.

Apparatus. See Experiment 1.
Procedure. Each participant underwent preexposure training on each of five days, with half of the participants training with centered frames (centered-frame group, $n=4$ ) and the other half training with offset frames (offset-frame group, $n=4$ ). The participants performed three blocks of trials each day, with proportions of trials in the first two blocks changing across the training sessions (Table 2). A new task, the edge task, was introduced and comprised the bulk of the training. During the first block of the edge task, the participants initially viewed an intact frame (centered or offset, depending on the preexposure group to which they were assigned) that subsequently appeared to dissolve after $1 \mathrm{sec}$, leaving only a [-fragment or ]-fragment. To influence the participants to think of the fragment as part of an intact frame, they were asked in these training trials to move a marker horizontally until it intersected the missing edge of the frame. Once the participants indicated their decision by pressing a key, feedback was provided in the form of a visual representation of the final marker location with respect to the real edge of the intact frame. An identical task was performed during a second edge task block, the only difference being that the fragment was presented without the initial fading intact frame, leaving participants to rely only upon their memory of the intact frame from previous trials to complete the task. Feedback was still provided, however, allowing the participants to view their responses with respect to the intact frame. After these two edge task blocks, participants then performed a midline task. This task was similar to that performed in Experiment 1; that is, participants were asked to move a marker to straight-ahead in the presence of the intact frames (centered or offset, depending on group assignment) or fragments (randomly ordered). However, in this task, trials now began with a blank screen, rather than with an initial vertical center line. In this midline task, participants received no feedback concerning performance accuracy.

\section{Results and Discussion}

In the edge task of the preexposure training sessions, the errors in locating the hidden edge of the frame decreased over the course of the subsequent sessions, as demonstrated by a significant effect of session $[F(1,550)=7.22$, $p<.05]$ in a two-way (session $\times$ group) ANOVA (Figure 4). This finding confirmed that the multiday training sessions allowed participants to better associate the visible fragment with the hidden remainder of the intact frame. To examine whether this additional training caused the Roelofs effect to be fully or partially mediated by a high-level representation of the remembered intact frame when the participant was shown only a fragment, we analyzed the pattern of responses from the midline task performed in the final block on the 5 th day of training, using a polarity $([$-fragment vs. ]-fragment) $\times$ position (left vs. middle vs. right) $\times$ group (centered-frame vs. offset-frame) repeated measures ANOVA (Figure 5). This analysis revealed a sig-

Table 2

Number of Trials Within Each Block During the Training Sessions of Experiment 2

\begin{tabular}{lccc}
\hline & $\begin{array}{c}\text { Edge Task } \\
\text { (Frame-Fading- } \\
\text { to-Fragment } \\
\text { With Feedback) }\end{array}$ & $\begin{array}{c}\text { Edge Task } \\
\text { (Fragments With } \\
\text { Feedback) }\end{array}$ & $\begin{array}{c}\text { Midline Task } \\
\text { (Frame and } \\
\text { Fragments; } \\
\text { No Feedback) }\end{array}$ \\
\hline Day 1 & 48 & 48 & 72 \\
Day 2 & 24 & 72 & 72 \\
Day 3 & 24 & 96 & 72 \\
Day 4 & 24 & 120 & 72 \\
Day 5 & 24 & 48 & 108 \\
\hline
\end{tabular}




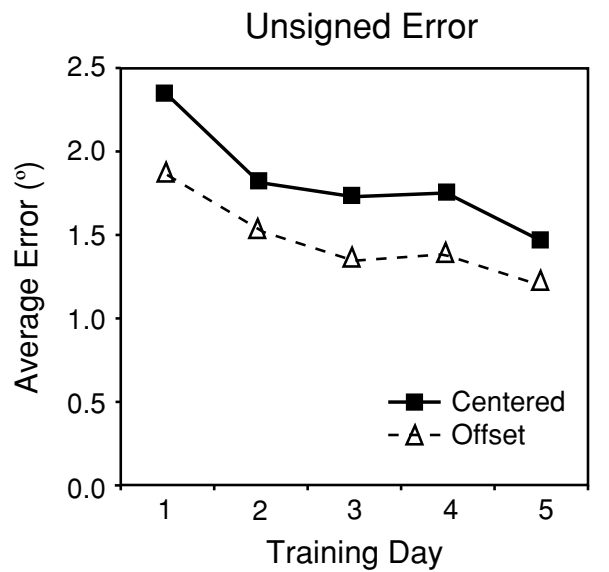

Figure 4. Effect of multiday training on the participants' accuracy in the edge task of Experiment 2, for the offset-frame and centered-frame groups. A significant main effect of session indicates successful training.

nificant effect of polarity and position (Table 3), replicating similar findings from Experiment 1. Notably, however, there was no significant main effect of group, nor any interaction involving group. The lack of these group effects, even with an effective training regimen spread over 5 days, suggests that the null effects found in Experiment 1 were not due solely to a lack of training efficacy and further demonstrates that the Roelofs effect is indeed driven by bottom-up processes and is not subject to change due to prior experience with different frames.

\section{EXPERIMENT 3}

The results of Experiment 1 yielded an unexpected finding: ]-fragments were found to induce a larger Roelofs effect than [-fragments. When expressed in this way, it would seem that this effect occurs in an allocentric or object-centered reference frame, with the right edge of a frame causing a larger effect than the left. However, it is a reasonable assumption that the participants were attending the marker for most of the trial duration (after all, their task was to adjust the marker's location so that it appeared to lie straight-ahead while simultaneously ignoring the frame). We can therefore further assume that they generally guided their fixation to the marker or a location nearby. Given this, the design of our stimuli would invariably place the ]- and [-fragments in the right and left visual fields, respectively. Thus, we propose that the differential effects of the two fragments were not based on allocentric coordinates but were instead driven by an asymmetry in the processing of images in the left versus the right visual fields.

The asymmetric effects of the ]- and [-fragments may be related to previous findings that the two visual hemifields are differentially represented in attentional processes. Several studies have found that the activations of the parietal cortices are predominantly right-lateralized during the reflexive orienting of attention (Corbetta, Miezin, Shulman, \& Petersen, 1993; Kim et al., 1999; Nobre et al., 1997). Lesions of the right parietal cortex very commonly lead to neglect of the left half of space, whereas left parietal lesions rarely lead to right hemineglect. Combined, these findings lead to a model in which the left parietal cortex has the ability to guide attention toward the right hemifield, whereas the right parietal cortex is capable of guiding attention to either hemifield (see Mesulam, 1999, for a review). This model then predicts that objects in the right visual field will be overrepresented by the brains of normal healthy observers. Indeed, healthy observers have been shown to display a small leftward bias when asked to bisect a horizontal line (a lesser version, known as pseudoneglect, of a symptom displayed in a more drastic form in neglect patients), as would be expected if the right half of the line were overestimated (Bowers \& Heilman, 1980; Jewell \& McCourt, 2000).

Some authors have suggested the possibility that the dominant role of the right parietal lobe in guiding attention is partly driven through an asymmetry in the neuro-

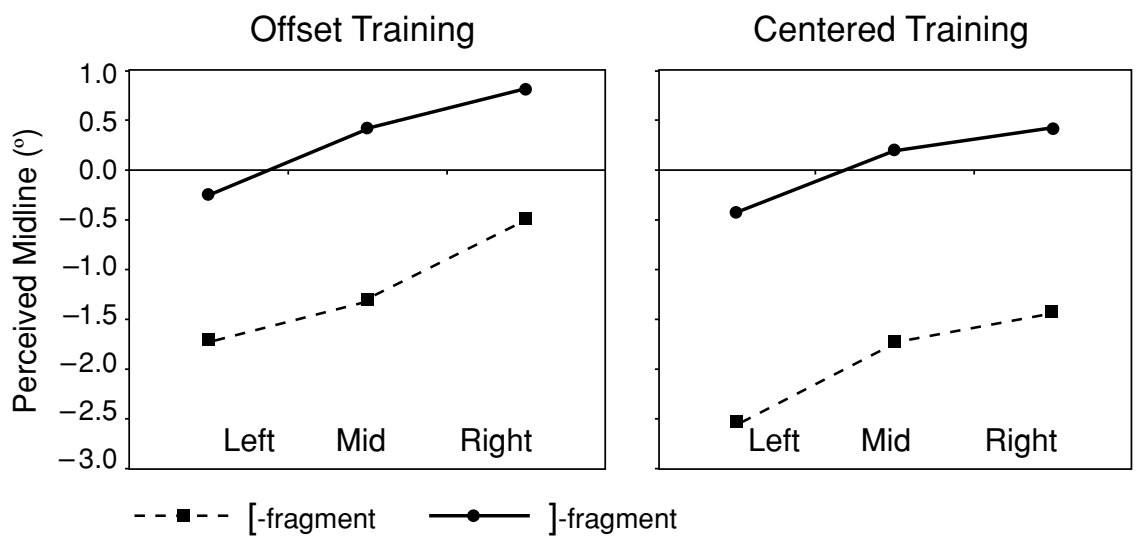

Figure 5. Distortions of the perceived midline in the offset-frame and centered-frame groups of Experiment 2, as measured after the fifth day of training. As in Experiment 1, there were no significant differences in the processing of the [- and ]-fragments across preexposure groups. 
Table 3

Experiment 2: Statistical Results

\begin{tabular}{llrc}
\hline \multicolumn{1}{c}{ Effect } & \multicolumn{1}{c}{$d f$} & $F$ & $p$ \\
\hline Polarity ([-fragment vs. ]-fragment) & 1,6 & 12.61 & $<.05$ \\
Position (left vs. middle vs. right) & 2,12 & 26.04 & $<.001$ \\
Group (offset vs. centered) & 1,6 & 0.39 & n.s. \\
Polarity $\times$ position & 2,12 & 0.42 & n.s. \\
Polarity $\times$ group & 1,6 & 0.24 & n.s. \\
Position $\times$ group & 2,12 & 0.72 & n.s. \\
Polarity $\times$ position $\times$ group & 2,12 & 0.31 & n.s. \\
\hline
\end{tabular}

nal projections of the subcortical structures (e.g., superior colliculus and pulvinar) known to be involved in attention and visual processing (Posner \& Cohen, 1980; Posner \& Rafal, 1987); however, others have shown that this effect seems to be modulated by eye dominance as well (Roth, Lora, \& Heilman, 2002). Specifically, evidence indicates that the afferent inputs to these subcortical structures from the retina are not uniform (Figure 6), with the nasal hemiretinas having a higher density of connections than the temporal hemiretinas (Hubel, LeVay, \& Wiesel, 1975; Pollack \& Hickey, 1979; Sherman, 1974; Sprague, 1966; Wilson \& Toyne, 1970; but see also Williams, Azzopardi, $\&$ Cowey, 1995). Thus, it is thought that these structures contribute different attentional effects depending on whether the image of the stimulus lands on the nasal or temporal hemiretinas. Indeed, researchers have noted that these subcortical anatomic asymmetries do yield observable behavioral differences. For example, the reflexive production of saccades has been found to be stronger for stimuli presented to the nasal hemiretina (Posner \& Cohen, 1980; Shulman, 1984), as has the attentional phenomenon of inhibition of return (Berger \& Henik, 2000; Rafal, Calabresi, Brennan, \& Sciolto, 1989). In addition, nonconscious priming has been found to be stronger for cues presented to the nasal hemiretina (Ansorge, 2003).

Here, we hypothesize that these same subcortical pathways might be mediating what appears to be the largely bottom-up effect of the Roelofs effect. If this is so, then patching one eye should cause differential effects for fragments that fall on the temporal versus nasal hemiretina, regardless of the viewing eye. Experiment 3 was designed to test this hypothesis and included eye dominance as a factor so that its influence on the behavioral outcome could be assessed as well. Furthermore, the data collected in Experiment 3 provided an additional opportunity to test the hypotheses put forth in Experiment 1 regarding the manner in which the effects of individual fragments can be combined to yield the Roelofs effect induced by an intact frame.

\section{Method}

Participants. The participants were University of Oregon students who gave informed consent and took part in the experiment in exchange for credit in an undergraduate psychology course. All were naive to the hypothesis and reported normal or corrected-tonormal vision.

Assessment of eye dominance. The participants first underwent two assessments of eye dominance. The first task began with the participants' fully extending the right arm and using the index finger to point at a small target on the wall under binocular viewing conditions. The participants were then asked to close one eye at a time and indicate which eye's view made the finger appear to jump away from the target (this indicated the nondominant eye). In the second test of eye dominance, the participants were asked to open both eyes and clasp their hands together (with arms extended) to form a telescope centered on the same target. Once the target was visible, they were asked to move the telescope slowly toward their face, always keeping the target visible in the center of the telescope, until their hands touched their face, surrounding the dominant eye. Of the 53 participants, 41 were right-eye dominant and 9 were left-eye dominant. The remaining 3 participants could not be consistently classified (i.e., they gave conflicting reports in the two dominance tests), and their data were therefore excluded from subsequent analyses.

Apparatus. See Experiment 1.

Procedure. The participants performed the experimental task using a paradigm identical to that used by the offset-frame group in the first experiment. However, the participants in the present experiment performed the task three times each: once with the right eye patched, once with the left eye patched, and once with both eyes open. The order of the eye patch conditions was randomized across the participants.

\section{Results and Discussion}

The results of Experiment 3 were initially analyzed with an ANOVA of viewing eye (left vs. right) $\times$ eye dominance (left vs. right) $\times$ polarity ([-fragment vs. ]-fragment $) \times$ position (left vs. middle vs. right). This analysis revealed significant main effects of polarity and position (Table 4), indicating that the fragments once again caused a significant Roelofs effect that was further modulated by the fragment's position. In addition, a significant interaction of polarity $\times$ position indicated that the ]-fragment caused

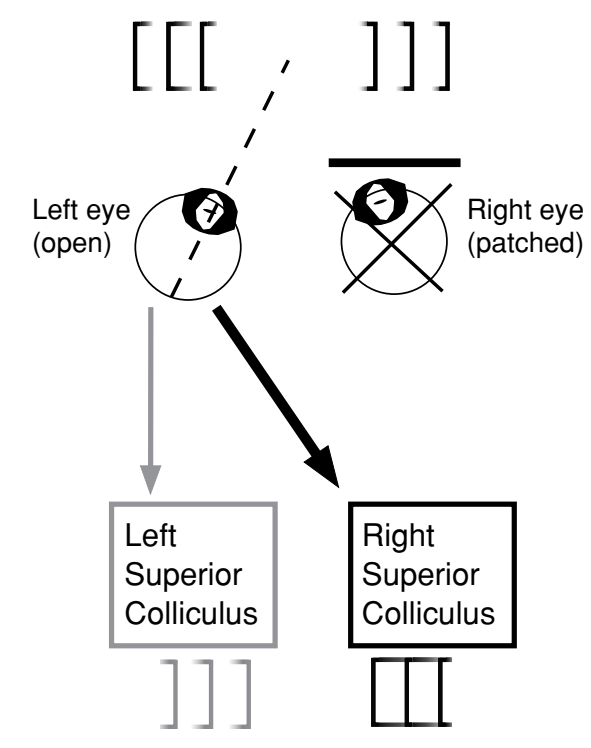

Figure 6. Schematic of the anatomical projections from the temporal and nasal hemiretinas to the superior colliculi. With the left eye open, fragments on the left side of space will project to the nasal hemiretina and have a relatively strong projection to the contralateral subcortical structures. Conversely, items on the right side of space will project to the temporal hemiretina and have a relatively weak projection to subcortical structures (denoted in lighter gray). Figure adapted from Ansorge (2003). 
Table 4

Experiment 3: Statistical Results

\begin{tabular}{lrrc}
\hline \multicolumn{1}{c}{ Effect } & $d f$ & $F$ & $p$ \\
\hline Coded by Fragment Polarity & & & \\
$\quad$ Viewing eye (left vs. right) & 1,48 & 1.29 & n.s. \\
Eye dominance (left vs. right) & 1,48 & 0.07 & n.s. \\
Polarity ([-fragment vs. ]-fragment) & 1,48 & 70.54 & $<.001$ \\
Position (left vs. middle vs. right) & 2,96 & 53.80 & $<.001$ \\
Polarity X position & 2,96 & 4.51 & $<.02$ \\
Coded by Hemiretina & & & \\
Viewing eye (left vs. right) & 1,48 & 2.63 & n.s. \\
Eye dominance (left vs. right) & 1,48 & 0.80 & n.s. \\
Hemiretina (nasal vs. temporal) & 1,48 & 1.29 & n.s. \\
Position (near vs. middle vs. far) & 2,96 & 53.17 & $<.001$ \\
Hemiretina X position & 2,96 & 0.40 & n.s. \\
\hline
\end{tabular}

a larger Roelofs effect than did the [-fragment (Figure 7). Notably, there were no significant effects of viewing eye or eye dominance.

To directly test the subcortical-mediation hypothesis, the fragment polarity was recoded in terms of the hemiretina (temporal or nasal) to which the image of the fragment projected when the participant was fixating on or near the marker location (e.g., the [-fragment projected onto the nasal hemiretina of the left eye when the right eye was patched, or the temporal hemiretina of the right eye when the left was patched, see Figure 6). ${ }^{3}$ In addition, fragment position was recoded to reflect the relative location of the fragment with respect to the participant's midsagittal plane (e.g., the right [-fragment was near the midline, whereas the left [-fragment was far from the midline). A subsequent ANOVA of viewing eye (left vs. right) $\times$ eye dominance (left vs. right) $\times$ hemiretina (nasal vs. temporal) $\times$ position (near to midline vs. middle vs. far from midline) revealed a significant main effect only of position (Table 4). The lack of a main effect for hemiretina suggests that the Roelofs effect is not mediated through subcortical structures with asymmetrical projections from the nasal and temporal hemiretinas.

To determine how the magnitude of the Roelofs effect from intact frames compared with that of the fragments,



Figure 7. Replicating the results of Experiment 1, fragments in the right visual field (]-fragments) caused a greater Roelofs effect than those in the left visual field ([-fragments), regardless of the viewing eye. we used the data from the block of trials in which both eyes viewed the visual display to once again test the predictions of the sum-of-its-parts, average-of-its-parts, outermostanchor, and innermost-anchor hypotheses that were described in Experiment 1. Paired $t$ tests indicated that the predictions of the average-of-its-parts, outermost-anchor, and innermost-anchor hypotheses were significantly different from the effect found with the intact frame $[t(52)=$ $6.73, p<.001 ; t(52)=2.47, p<.05 ; t(52)=3.78, p<$ .001 , respectively]. However, the predictions of magnitude based on the sum-of-its-parts hypothesis did not significantly differ from the magnitude of the Roelofs effect induced with intact frames $[t(52)=1.032$, n.s.], indicating that this hypothesis once again adequately described the effect found with the intact frame.

\section{GENERAL DISCUSSION}

\section{Bottom-Up Versus Top-Down Processing}

We did not find any effect of prior experience in our tasks, suggesting that, in general, immediately visible information drives the Roelofs effect in a bottom-up fashion more robustly than do top-down (and invisible) elements. That is, the visible fragments produced shifts of the apparent midline in accordance with their distance from the observer's midsagittal plane (i.e., a distant fragment produced larger shifts of apparent midline than did a closer one), rather than as a result of the locations of the (partially occluded) frames that they represented.

In contrast to this finding, other studies have demonstrated that experience with a particular stimulus set can bias the manner in which observers perceive a partially occluded object. Zemel, Behrmann, Mozer, and Bavelier (2002) found that preexposure to an intact stimulus in one block of trials would bias the manner in which objectbased attention was distributed to the different visible fragments of the object when it was shown partially occluded in a subsequent block of trials. In another study that examined the effects of experience on the perception of partially occluded objects, Joseph and Nakayama (1999) asked observers to judge the direction of motion of a pair of objects undergoing ambiguous apparent motion. Although the shape of the unoccluded objects caused a bias in the observer's perception of their motion, the addition of the occluder caused the perception to be unbiased. However, a brief preexposure to the stationary unoccluded objects before the addition of the occluder was enough to bias the observer's perception of the subsequent apparent motion. Unlike Zemel et al., however, Joseph and Nakayama found this effect to be short-lived, with the bias disappearing 2-6 sec after the preexposure period.

While it is clear that the present results indicate that the Roelofs effect is not subject to a long-lasting, top-down modulation like that found by Zemel et al. (2002), it is possible that a short-lived modulation like that found by Joseph and Nakayama (1999) went undetected. For this reason, it is useful to speculate on why the durations of the top-down effects differed so drastically in the studies of Zemel et al. and Joseph and Nakayama. First, the two stud- 
ies were using two different types of amodal representation. In the study of Zemel et al., both ends of the stimuli were visible, with the occluder covering just the middle portion of the object so that representation of the whole object was accomplished through amodal completion. In contrast, the occluder in the study of Joseph and Nakayama completely covered one end of the stimulus, so that the representation of the whole could be accomplished only through amodal continuation. It may be that the brain mechanisms of amodal completion and those of continuation differ in a way that leads to different time courses, with completion having long-term effects on perceptual processing and continuation having short-term effects. It should be noted that the stimuli of the present study required amodal continuation for the formation of a representation of the intact frame based on the presentation of a single fragment. Thus, if amodal continuation truly is immune to long-term effects of preexposure, it is possible that the null effects of training in our experiments were caused by too long an interval between the preexposure period and the eventual fragment presentations.

A second difference in the studies of Zemel et al. (2002) and Joseph and Nakayama (1999) lies in the ongoing relationships between the preexposure stimuli and the eventual occluded fragments. In Zemel et al.'s paradigm, each participant experienced only a single preexposure stimulus for each of the partially occluded stimuli presented later. In contrast, Joseph and Nakayama changed this relationship from trial to trial, with each participant experiencing, over the course of the experiment, two possible preexposure stimuli for each subsequent partially occluded stimulus. This would have certainly contributed to the short duration of their measured preexposure effects. However, this could not account for the lack of long term preexposure effects in the present study, since, as in the study of Zemel et al., our participants experienced a one-to-one relationship between the preexposure stimuli and the corresponding fragments.

A third possible explanation for the different durations of the preexposure effects in the studies of Zemel et al. (2002) and those of Joseph and Nakayama (1999) lies in the differences in the way these effects were measured. Zemel et al. measured the effects of preexposure on the manner in which object-based attention was distributed across the partially obscured object. In contrast, Joseph and Nakayama measured the effects of preexposure on the participant's perception of ambiguous apparent motion. It might be that preexposure simply affects different brain processes with different time courses. If this is the case, it is also possible that preexposure can have only a shortlived impact on the Roelofs effect, so that it remained undetected in the present study. Ongoing studies in the lab are attempting to assess whether this is the case.

\section{From Fragments to Intact Frames}

In order to more fully characterize these bottom-up processing effects, we tested a number of hypotheses regarding the manner in which the fragment effects related to the effects of the intact frame. The hypothesis that best fit the relationship was the sum-of-its-parts explanation-that is, the magnitude of the Roelofs effect seen with an intact frame roughly equaled the sum of the effects found with the corresponding [- and ]-fragments. This model explained the data better than did another hypothesis based on an average of the [- and ]-fragment effects, or the hypotheses that either the most peripheral or most central fragments acted as anchors in causing the effect found with the intact frame. The sum-of-its-parts explanation is interesting in light of a recent study by Li and Matin (2005) that also investigated the relationship of parts to the whole in a related illusion, the rod-in-frame illusion. In the rod-in-frame illusion, a small rod is presented in the context of a large, square frame that is tilted off vertical. The surrounding frame induces a distortion of perceived vertical, thus causing a misperception of the orientation of the center rod. Li and Matin found that the effect of the entire tilted frame was less than the combined effects of the individual frame parts presented in isolation. In contrast, our data suggest that the Roelofs illusion can be explained quite well as a sum of the effects from the two ends of the frame. The discrepancy in the relationship of parts to the whole suggests that although illusion magnitudes for the rod-in-frame and Roelofs effect are correlated within observers (Dassonville, Walter, \& Lunger, 2006), their different mechanisms (tilting the perceived vertical vs. pulling the perceived midline) likely utilize visual context in different ways. Thus presenting only a portion of the illusory context will have different effects in the two cases.

\section{Visual Field Asymmetry}

Finally, the present results demonstrate an interesting visual field asymmetry, in which fragments presented in the right visual field tended to cause a larger Roelofs effect than did those presented in the left visual field. This effect seems analogous to pseudoneglect, in which healthy subjects incorrectly bisect a horizontal line due to an overestimation of the length of the line extending rightward (Bowers \& Heilman, 1980; Jewell \& McCourt, 2000). It seems plausible, then, to suggest that the causes of the two phenomena are the same. Previous work on pseudoneglect (Bowers \& Heilman, 1980; Jewell \& McCourt, 2000) and the neglect associated with lesions to the parietal cortex suggest that the right visual field is doubly represented within the attentional systems of the brain, with the parietal cortex of the left hemisphere capable of guiding attention toward the right, and the right parietal cortex capable of guiding attention in either direction (Mesulam, 1999). However, this left-right asymmetry in the Roelofs effect does not seem to be driven by differences in the anatomical connections of the nasal and temporal hemiretinas, since it was present whether the subject was viewing the stimulus through the left eye, the right eye, or both eyes.

\section{REFERENCES}

Ansorge, U. (2003). Asymmetric influences of temporally vs. nasally presented masked information: Evidence for collicular contributions to nonconscious priming effects. Brain \& Cognition, 51, 317-325.

Berger, A., \& HeniK, A. (2000). The endogenous modulation of IOR 
is nasal-temporal asymmetric. Journal of Cognitive Neuroscience, 12, 421-428.

Bowers, D., \& Heilman, K. M. (1980). Pseudoneglect: Effects of hemispace on a tactile line bisection task. Neuropsychologia, 18, 491-498.

Brecher, G. A., Brecher, M. H., Kommerell, G., SAuter, F. A., \& SellerbeCK, J. (1972). Relation of optical and labyrinthean orientation. Optica Acta, 19, 467-471.

Bridgeman, B., Peery, S., \& ANand, S. (1997). Interaction of cognitive and sensorimotor maps of visual space. Perception \& Psychophysics, 59, 456-469.

Brosgole, L. (1968). An analysis of induced motion. Acta Psychologica, 28, 1-44.

Corbetta, M., Miezin, F. M., Shulman, G. L., \& Petersen, S. E. (1993). A PET study of visuospatial attention. Journal of Neuroscience, 13, 1202-1226.

Coren, S., \& Porac, C. (1987). Individual differences in visualgeometric illusions: Predictions from measures of spatial cognitive abilities. Perception \& Psychophysics, 41, 211-219.

Dassonville, P., \& Bala, J. K. (2004a). Are the original Roelofs effect and the induced Roelofs effect confounded by the same expansion of remembered space? Vision Research, 44, 1025-1029.

Dassonville, P., \& Bala, J. K. (2004b). Perception, action, and Roelofs effect: A mere illusion of dissociation. PLoS Biology, 2, 1936-1945 (e364).

Dassonville, P., Bridgeman, B., Bala, J. K., Thiem, P., \& SamPANES, A. (2004). The induced Roelofs effect: Two visual systems or the shift of a single reference frame? Vision Research, 44, 603-611.

Dassonville, P. Walter, E., \& Lunger, K. A. (2006). Illusions of space, field dependence and the efficiency of working memory. Journal of Vision, 6, 476.

de Grave, D. D., Brenner, E., \& Smeets, J. B. J. (2002). Are the original Roelofs effect and the induced Roelofs effect caused by the same shift in straight ahead? Vision Research, 42, 2279-2285.

HARRIS, C. S. (1974). Beware of the straight-ahead shift-a nonperceptual change in experiments on adaptation to displaced vision. Perception, 3, 461-476

Hubel, D. H., LeVay, S., \& Wiesel, T. N. (1975). Mode of termination of retinotectal fibers in macaque monkey: An autoradiographic study. Brain Research, 96, 25-40.

Jewell, G., \& McCourt, M. E. (2000). Pseudoneglect: A review and meta-analysis of performance factors in line bisection tasks. Neuropsychologia, 38, 93-110.

JOSEPH, J. S., \& NAKAYAMA, K. (1999). Amodal representation depends on the object seen before partial occlusion. Vision Research, 39, 283-292.

Karni, A., Tanne, D., Rubenstein, B. S., Askenasy, J. J., \& Sagi, D. (1994). Dependence on REM sleep of overnight improvement of a perceptual skill. Science, 265, 679-682.

Kim, Y. H., Gitelman, D. R., Nobre, A. C., Parrish, T. B., LaBar, K. S., \& Mesulam, M. M. (1999). The large-scale neurological network for spatial attention displays multifunctional overlap but differential asymmetry. NeuroImage, 9, 269-277.

LEE, D. N., \& ARONSON, E. (1974). Visual proprioceptive control of standing in human infants. Perception \& Psychophysics, 15, 529-532.

LI, W., \& Matin, L. (2005). The rod-and-frame effect: The whole is less than the sum of its parts. Perception, 34, 699-716.

MacDougall, R. (1903). The subjective horizon. Psychological Monographs, 4, 145-166.

Matin, L., \& Fox, C. R. (1989). Visually perceived eye level and perceived elevation of objects: Linearly additive influences from visual field pitch and from gravity. Vision Research, 29, 315-324.

Mesulam, M. M. (1999). Spatial attention and neglect: Parietal, frontal and cingulate contributions to the mental representation and attentional targeting of salient extrapersonal events. Philosophical Transactions of the Royal Society of London: Series B, 354, 1325-1346.

Nobre, A. C., Sebestyen, G. N., Gitelman, D. R., Mesulam, M. M., Frackowiack, R. S. J., \& Frith, C. D. (1997). Functional localization of the system for visuospatial attention using positron emission tomography. Brain: A Journal of Neurology, 120, 515-533.

Pollack, J. G., \& Hickey, T. L. (1979). The distribution of retinocollicular axon terminals in rhesus monkey. Journal of Comparative Neurology, 185, 587-602.
Posner, M. I., \& Cohen, Y. (1980). Attention and the control of movements. In G. Stelmach \& J. Requin (Eds.), Tutorials in motor behavior (pp. 243-258). Amsterdam: Elsevier, North-Holland.

Posner, M. I., \& Rafal, R. D. (1987). Cognitive theories of attention and the rehabilitation of attentional deficits. In M. J. Meier, A. L. Benton, \& L. Diller (Eds.), Neuropsychological rehabilitation (pp. 182201). Edinburgh: Churchill Livingstone.

Rafal, R. D., Calabresi, P. A., Brennan, C. W., \& Sciolto, T. K. (1989). Saccade preparation inhibits reorienting to recently attended locations. Journal of Experimental Psychology: Human Perception \& Performance, 15, 673-685.

Roelofs, C. (1935). Optische Lokalization. Archive für Augenheilkunde, 109, 395-415.

Roth, H. L., Lora, A. N., \& Heilman, K. M. (2002). Effects of monocular viewing and eye dominance on spatial attention. Brain, 125, 2023-2035.

Sherman, S. M. (1974). Visual fields of cats with cortical and tectal lesions. Science, 185, 355-357.

Shulman, G. L. (1984). An asymmetry in the control of eye movements and shifts of attention. Acta Psychologica, 55, 53-69.

SpRAGUe, J. M. (1966). Interaction of cortex and superior colliculus in mediation of visually guided behavior in the cat. Science, 153, 15441547.

Stickgold, R., Whidbee, D., Schirmer, B., Patel, V., \& Hobson, J. A. (2000). Visual discrimination task improvement: A multi-step process occurring during sleep. Journal of Cognitive Neuroscience, $12,246-254$.

WeRner, H., WAPNER, S., \& BRUELL, J. (1953). Experiments on sensorytonic field theory of perception: VI. Effect of position of head, eyes, and of object on the position of the apparent median plane. Journal of Experimental Psychology, 46, 293-299.

Williams, C., Azzopardi, P., \& Cowey, A. (1995). Nasal and temporal retinal ganglion cells projecting to the midbrain: Implications for "blindsight." Neuroscience, 65, 577-586.

WiLson, M. E., \& ToYNE, M. J. (1970). Retino-tectal and cortico-tectal projections in Macaca mulatta. Brain Research, 24, 395-406.

WitKIN, H. A., \& Asch, S. E. (1948). Studies in space orientation. IV. Further experiments on perception of the upright with displaced visual fields. Journal of Experimental Psychology, 38, 762-782.

Zemel, R. S., Behrmann, M., Mozer, M. C., \& Bavelier, D. (2002). Experience-dependent perceptual grouping and object-based attention. Journal of Experimental Psychology: Human Perception \& Performance, 28, 202-217.

\section{NOTES}

1. A similar distortion of the subjective midline is manifest when the entire visual scene is displaced to the left or right through the use of prism lenses, even without the sensorimotor adaptation that occurs when the observers are provided feedback about the accuracy of their goal-directed movements (Harris, 1974). In many ways, the Roelofs-inducing frame is similar to the displaced visual scene that is obtained with prism lenses, and undoubtedly the associated distortions of the subjective midline are related (and perhaps even identical). Because of this, it is possible that many, if not all, of the findings reported in the present study would apply equally to either condition. However, since the present experiments make use of a stimulus similar to that of Roelofs (Roelofs, 1935), we will refer to the observed perceptual distortion as the Roelofs effect.

2. There is, however, some dispute about whether the original and induced Roelofs effects are generated by identical mechanisms (Dassonville \& Bala, 2004a; de Grave, Brenner \& Smeets, 2002).

3. The accuracy of this recoding is dependent upon the assumption that the participant generally fixated on or near the marker, as opposed to, for example, the location of the fragment (which the participant was instructed to ignore). However, since the participant was charged with the relatively difficult task of moving the marker until it appeared to lie straight-ahead in a darkened environment, this assumption seems reasonable.

(Manuscript received June 22, 2005; revision accepted for publication November 23, 2005.) 